\title{
DE KOERLANDSE KOLONISATIEPOGINGEN OP TOBAGO
}

DOOR

\section{Dr. J. Kleyntjens S.J.}

Het succesvol optreden van sommige landen bij de verwerving van overzees gebied en de belangrijke rol welke zij zich daardoor in de zeventiende-eeuwse wereldhandel zagen toebedeeld, hebben ertoe geleid, dat men zich hier en daar een iet wat overdreven voorstelling is gaan maken van de waarde welke uit een commercieel oogpunt aan een dergelijk bezit moet worden toegekend. Een wel zeer merkwaardig voorbeeld van een uit dien hoofde gevoerde kolonisatiepolitiek is de nog al eens onderbroken activiteit van de hertogen van Koerland met betrekking tot het ten noordoosten van Trinidad gelegen eiland Tobago, een der kleine Antillen.

Dat Columbus dit eiland zou hebben ontdekt, vindt vrijwel geen serieuse aanhang meer; dat het Robinson Crusoë tot verblijf zou hebben gediend is weinig aannemelijk, gezien het feit dat in de dagen, waarin het prototype voor Defoe's romanfiguur moet hebben geleefd Tobago reeds door blanken werd bewoond. Aanvankelijk heeft ook dit eiland binnen de Spaanse invloedssfeer gelegen, doch de eerste meer doeltreffende vestigingspogingen schijnen op Engels en Hollands initiatief te zijn ondernomen. Hoe dit echter ook zij, vaststaat in ieder geval, dat Karel II Tobago op 17 November 1664 aan hertog Jacobus van Koerland heeft afgestaan. De hertog had vorderingen op de Engelse Kroon, en bovendien was de Tweede Engelse oorlog met de Republiek in het zicht, om welke reden Karel het wenselijk achtte, dat Tobago in handen kwam van een neutrale souverein. Herhaaldelijk is door hertog Jacobus en zijn opvolgers getracht het eiland te koloniseren, af en toe in combinatie, vaak ook in concurrentie, met de Hollanders die zich te dien einde op een ouder, huns inziens beter, recht beriepen. De verschillende expedities zijn vooral door W. R. Menkman in De West-Indische Gids uit de 
jaren 1939 en 1940 beschreven ${ }^{1}$ ), hoofdzakelijk aan de hand van de gedrukte bronnen en enkele stukken uit het Rotterdamse Gemeentearchief 2). Wat echter meer speciaal de Koerlandse aanspraken betreft ligt het voor de hand, dat daarover in de archieven nog wel iets te vinden zal zijn. Het aanstonds nog nader te noemen proces, dat destijds te 's Gravenhage vermoedelijk voor het Hof van Holland tussen de hertog en de Zeeuwse kolonisatiecompagnie van de gebroeders Lampsins is gevoerd, heeft natuurlijk zijn papieren omslag meegebracht, waarvan dan een en ander tezamen met de overige stukken van het Hof op het Algemeen Rijksárchief terecht moet zijn gekomen. Voorts zij nog gewezen op de archieven van de voormalige West-Indiese Compagnie, alsmede op een andere collectie van op West Indië betrekking hebbende stukken, welke zich blijkens „,The General States Archives and their Contents", 1932, p. 14, in dezelfde bewaarplaats bevinden.

Dient dit alles nog nader te worden onderzocht, de Koerlandse archieven in Riga laten ons wat dat betreft zeer zeker niet in de steek. Tijdens mijn verblijf aldaar in de jaren vóór de oorlog ontdekte ik op het Staatsarchief in Riga een in het Frans gesteld manuscript van Ewald baron von Klopmann, getiteld: „Abrégé de l'Histoire de Tobago" met enkele bijlagen, aan welke stukken tot heden toe te weinig aandacht is besteed.

Von Klopmann, die in 1734 in Würzau (Koerland) geboren werd, heeft na het beëindigen van zijn studie aan de universiteit van Jena verschillende Europese landen bereisd. In 1760 keerde hij naar zijn vaderland terug, waar hij tot de dienst aan het Hof geroepen werd. Na de ineenstorting van het hertogdom in 1795 vestigde hij zich in Mittau, waar hij ten jare 1804 overleed. Als hoofd van de hertogelijke archieven en bibliotheek stonden hem de bronnen ter beschikking, waaruit hij voor de samenstelling van zijn overzicht heeft kunnen putten. En daar hij, zoals hijzelf mededeelt, met het oog op de zaken van Tobago door de hertog met een zending naar Engeland is belast geweest, was er voor hem reden te over om zich dienaangaande uitvoerig te documenteren. Ligt het daarom min of meer voor de hand, dat hij in zijn

1). Resp. p. 218 e.v. en p. 33 e.v. Aan deze artikelen werd voor het bovenstaande het een en ander ontleend.

2) Vergelijk in dit verband nog: „,Stukken betrekkelijk het eiland Tobago 1664-1684-1698" door W. R. Menkman (Rotterdamsch Jaarboekje, 1939, p. 80). 
memorie de rechten van de hertogen van Koerland op hun voordeligst zal hebben voorgesteld, zodat het niet is uitgesloten, dat het stuk voor wat dat betreft enigermate als een partijstuk moet worden beschouwd en dientengevolge met omzichtigheid dient te worden gehanteerd, dat neemt niet weg, dat men zich voor wat de opsomming der blote feiten aangaat bij het oordeel van Otto Heinz Mattiesen kan aansluiten, die het in zijn in 1940 verschenen werk: „Die Kolonial- und Ubersee-Politik der Kurländischen Herzöge im 17 und 18 Jahrhundert”, p. 436 betreurt, dat „,Klopmann's nüchterne Feststellungen am wenigsten berücksichtigt wurden".

$\mathrm{Nu}$ Mattiesen het relaas van von Klopmann in zijn evengemeld werk weliswaar heeft benut, doch niet heeft afgedrukt, en het bovendien allerminst is uitgesloten, dat dit stuk het lot van het grootste gedeelte van het Lettische Staatsarchief zal hebben gedeeld en door de Duitsers of Russen is weggesleept of verbrand ${ }^{1}$ ) is publicatie van dit rapport, dat ook een belangrijke bijdrage levert tot de kennis van het Nederlandse aandeel in de ,,schipvaert" op de West in vroeger eeuwen, van dubbel groot belang. Vormt het bovendien een welkome aanvulling en menigmaal een correctie op de publicaties van Menkman, anderzijds laat het talrijke door Menkman vermelde byzonderheden weg, hetgeen weer steun geeft aan mijn bovenvermelde zienswijze, dat het voor bepaalde doeleinden werd geschreven en uit de koloniale geschiedenis van Koerland slechts de daartoe bruikbare aspecten naar voren brengt.

Laat Menkman de relaties tussen de Koerlandse hertog en de Engelse Kroon reeds onder de regering van Jacobus I een aanvang nemen, het rapport begint met de stand van zaken tijdens het bewind van Karel I. Menkman spreekt al van Koerlandse kolonisatiepogingen uit de jaren 1634 en 1642, de laatste onder leiding van Cornelis Caron. Het rapport noemt geen van beide, doch vermeldt uitsluitend die van 1654 met het schip de ,Hertogin van Koerland" onder bevel van de Nederlander Willem Mollens. Dit is dus de expeditie welke volgens Menkman samenviel met die van de gebroeders Lampsins uit Vlissingen, en die, anders dan Menkman schijnt te veronderstellen, allerminst zonder succes verlopen is. In de volgende jaren toch werden herhaaldelijk nog schepen, voor Koerlandse rekening uitgerust, naar Tobago

1) Zie mijn artikel in „Archives, Bibliotheques” (Brussel) December 1948. (Het Ms. berustte in het Valtsarchief te Riga no. 250). 
gezonden en eerst de gevangeneming van de hertog door de Zweedse generaal Douglas was volgens het rapport oorzaak, dat de Zeeuwen zich zogenaamd uit voorzorg en met de voorgewende bedoeling het later integraal terug te geven van het Koerlandse fort en onderhorigheden op Tobago meester makkten ${ }^{1}$ ).

Een ander punt, waarop Menkman blijkbaar door het rapport wordt gecorrigeerd, is de dagtekening van het proces, dat na de vrijlating van de hertog en in verband met de opgemelde occupatie van het Koerlandse fort door de Zeeuwen hier te lande is gevoerd. Menkman noemt het jaar 1671, de rapporteur plaatst dit proces veel eerder, nog vóór de zending van Von Dräckewitz naar Engeland in Maart 1660. Voorts is Menkman nog in zoverre onjuist, als hij de vertegenwoordiger van de hertog bij dit proces met de naam van Peter von Volckershoven aanduidt, terwijl in het rapport van Melchior Folckersamb sprake is 2).

Van de door Menkman vermelde mislukte tocht van 1675 en het afwijzend oordeel van de Engelse koning met betrekking tot Jacobus' aanspraken wordt - ik zou haast zeggen uiteraard generlei melding gemaakt. Slechts wordt het verlies van „De Blompot" onder kapitein Nagel gerelateerd, welk schip door Algerijnse zeerovers werd opgebracht. Het merkwaardige is hier, dat aan de meegezonden en voor de gouverneur van Barbados bestemde order van de Engelse koning, die Menkman op 19 Januari 1679/80, het rapport op 19 Februari 1680, dateert, niet de door Menkman gewraakte interpretatie gegeven wordt, als zoude deze naast een permissie aan de Koerlandse schepen om op Barbados te approvianderen ook een aanbeveling van de onderneming zelf hebben ingehouden. Nieuw is voorts nog het bericht omtrent het naar aanleiding van de molestatie van ,De Blompot" door de Sultan verstrekte vrijgeleide.

1) Over de agent van Jacobus, aan wie deze tijdens zijn gevangenschap de belangen der hertogelijke kolonien had toevertrouwd, zie mijn opstel „Kurzemes hercogs un Abrams Vikförs" in ,'Turpinäjums sekos", Riga, 1938, p. 566 e.v. en Latvijas un Hollandes attiecibas no. 13 lüdz 19 gadsimtam" 1936.

2) Het is altijd nog een open vraag, of er feitelijk wel een proces gevoerd is. Het optreden van Folckersamb, dat Menkman (De West Indische Gids, 1939, p. 312) op 1671 stelt, viel, zoals uit het rapport blijkt, in ieder geval veel vroeger. Wellicht kwamen Menkman of zijn zegsman tot hun datering op grond van het bestaan van de ,,Déduction par laquelle il est clairement monstré que l'isle de Tobago, sise en l'Ame- 
In tijdsorde volgen 1) daarna nog de tochten van kolonel Monck, kapitein John Poyntz en Dietrich von Alten Bockum. Chronologisch zou deze laatste zeer wel de gouverneur geweest kunnen zijn, die volgens Menkman in 1686 op het eiland is overleden. Zijn vertrek daarheen wordt door Von Klopmann op 1684 gesteld, zodat de aankomst moeilijk, zoals Menkman beweert, in 1687 kan hebben plaatsgehad.

Ook bij de zendingen van Von Blomberg en Praetorius, die ten doel hadden in Engeland belangstelling te wekken voor de onderbroken kolonisatiepogingen wordt uitvoerig stilgestaan, terwijl wij voor het geldend maken van de Koerlandse aanspraken gedurende de latere jaren uitsluitend op het rapport zijn aangewezen. Al met al dus reden genoeg om dit stuk door de druk openbaar te maken.

\section{Abrégé de 'L Histoire de TABAGo \\ par Ewald von Klopmann}

L'isle de Tabago, acquise par le Duc Jacques de Courlande sous Charles I, roi d'Angleterre est une des Antilles septentrionales dans la mer du Nord. Très fertile, ayant des plantations de sucre, de tabac, de café et même de muscade. Elle abonde en rivières au nombre de 18 , qui traversent le pays et offrent des communications propres à faciliter le commerce; elle a plusieurs excellents ports, pouvant même abriter des vaisseaux de ligne et douze baies, à savoir celles de:

1. Jacobus avec le Fort. 2. Ferdinand. 3. les Soeurs et frères. 4. Courlande. 5. Bonne Espérance. 6. Windau. 7. Petit Tabago. 8. Frederic. 9. Casimir. 10. Sable. 11. Kannoi. 12. Palsmitten.

ricque, appartient a Monseigneur le Duc en Livonie de Courlande et Semgalle. Et que la possession que Mrs les Lambsons prétendent sur la dite isle n'a aucun fondement. Imprimé l'an de grace 1668 à Mytaw, chez Michel Karnall (une autre édition de 1676, 24 pag., français et hollandais, l'édition de 1676 contient $1^{\circ}$ p. 1-16 la réimpression du manifeste de 1668 , imprimé à Mitaw (Koerland) $2^{\circ}$ p. 16-22 nouveau appendix à ce manifeste).

1) In zijn ,,Abrégé” beschrijft v. Klopmann nauwkeurig de byzonderheden waarmede de expedities van uit Windau in Koerland plaats hadden, de overtocht over den Oceaan en de aankomst te Tobago, waarvoor aan v. K. de ,Scheepsboeken”, (die in 1938 nog in het archief te Riga aanwezig waren) ten dienste stonden. 
Sa longueur est de huit milles allemandes ${ }^{1}$ ); la largeur est presque la même, le Duc Jacques l'a conquise sous le règne de Charles I, roi d'Angleterre et Oliver Cromwell 2) lui accorda en 1651 une charte, par laquelle il lui permettait la libre entrée dans tous les ports du Royaume, ne payant que la douane des vaisseaux de la nation. Le Duc encouragé par une aussi généreuse concession, envoya plusieurs bâtiments à Tabago avec des colonistes Courlandais pour peupler l'île. Il y fit bâtir un fort, qui porta son non ,,Jacobus". Le premier vaisseau, qu'il y envoya, pour faire des plantations après la découverte faite, fut nommé „,Duchesse de Courlande" 3) et fut commandé par Guillàume Mollens 4).

Avec 120 hommes d'équipage, il jeta l'ancre a l'isle de Tabago le 20 mai 1654 et l' ayant trouvée déserte, couverte d'épaisses forêts, il en prit possession pour le Duc, son Mâitre, selon la coutume générale, selon laquelle les îles du nouveau-monde sont subjugées. Mollens y dressa des colonies de différentes nations, y distribua assez d'arpents de terre pour y faire de considérables plantations. L'année suivante, 1655 au mois d'août, le Duc envoya un second vaisseau conduit par Jean Brand s), pour y porter des rafraîchissements et des munitions et la même année, un autre bâtiment avec 130 hommes d'équipage, bâtiment nommé

la Constance" ,commandé par Wolfart de Brederot, nommé

1) Het eiland Tobago is $42 \mathrm{~km}$ lang en $12^{1 / 2} \mathrm{~km}$ breed met een oppervlakte van $295 \mathrm{~km}^{2}$ cf: I. C. Praetorius: Tobago insulae Caraibicae in America sitae fatum. Groningae 1727, blz. 1.

2) Cromwell had op dit tijdstip weinig belangstelling voor de koloniale kwesties, zodat hertog Jakob alle vrijheid van handelen had. Daar de Engelse kolonies gedeeltelijk zonder controle waren, kon Jakob zich gemakkelijk van Tobago meester maken. Toen de Nederlands-Engelse oorlog uitbrak, bezette Jacob de territories in Afrika. Hij kon op den steun van Frankrijk rekenen.

3) Otto Heinz Mattiesen: Die Kolonial- und Ueberseepolitik der Kurländischen Herzöge im 17en und 18en Jahrhundert 1940 noemt het schip ten onrechte ,,Wapen van de hertogin van Koerland”.

4) Willem Mollens, Hollander van geboorte, was de eerste Kapitein, die in naam van hertog Jacobus van Koerland, het eiland Tobago in bezit nam. Later vertrok hij naar Jamaica. Over de kolonisatieplannen van den hertog-koopman Jacob, cf: Sitzungsberichte der Kurländischen Gesellschaft für Lit. und Kunst 1878, p. 68-70; Mitteilungen aus der livländischen Geschichte XII, 397; XIII, p. 49. I. Kleyntjens: Kurzemes hercogs Jekabs un Abrams Vikfōrs (zeme 1938 p. 566-577). Hamelberg: De Nederlanders op de W.I. eilanden. J. Skutch: Die Inseln Trinidad und Tobago (1929) waarin echter bijna alle Baltische publicaties over Tobago ontbreken.

s) Jan Brand, cf: Mattiesen o.c. p. 465. 
Clothing ${ }^{1}$ ) et qui fut nommé ensuite Directeur de l'île. Le vaisseau fut suivi d'un autre de $120 \mathrm{~h}$. d'équipage ayant à leur tête André Bohm. L'année 1657, le Duc fit expédier encore un vaisseau, accompagné d'une galliote, nommé ,,la Duchesse" 2) commandée par Nicolas de Brien et chargée de vivres et de munitions. Mollens, revenu de son premier voyage, fit son rapport au Duc qui fut très content de lui; il retourna à Tabago en 1658, au mois d' aôut, sur le bàtiment ,le Cavalier" pour y porter de réchef des vivres et des munitions. Au mois d'octobre de la même année, le Duc fit occuper de nouveau un vaisseau avec 150 hommes, ,"le roi David", commandé par Jacques Albert, avec vivres munitions, ustensiles; à ce vaisseau succéda un autre nommé ,,le Islande" 3) sous le commandement de Jeble Hiller, avec 100 hommes d'equipage et qui fit voile sur Lubeck, pour y prendre le reste de sa charge. Mais le malheur arrivé au Duc, le 10 octobre 1658 , si fatal à lui et à sa famille, interrompit les progrès de l'exécution de son plan. Le Duc fut enlevé la nuit de son château, à Mitau, avec toute sa famille, par Mr. Douglas, général Suédois, d'une manière peu convenable au ,,droit des gens" ayant observé dans toutes ses procédures à lui la plus exacte neutralité et reçu même dés assurances satisfaisantes du dit Général de n' avoir rien à craindre de sa part. - Enfin, ils furent tous conduits à Narva, par Riga et Ivangorod et ils y restèrent jusqu' apres la paix d'Oliva, 23 avril 1660, tous les Ministres des cours etrangères s'intéressant au sort du Duc et de sa famille 4).

Pendant cet intervalle, une Compagnie Zélandaise, des gens riches et entreprenants, du nom d'Adrien et Corneilie Lampsen s),

1) Jan Wolfert van Brederode, heer van Cloetingen (niet: genaamd van Clothing, zoals ook Mattiesen schrijft) was een Zeeuws edelman.

2) De galliote heette ,la Duchesse", niet het schip, zoals Mattiesen meent (o.c. 468).

3) Niet (zoals bij Mattiesen 469) ,,Der Isländer”. De vaart duurde van 10 Juni 1656 tot 8 Oct. 1657. (Archief Riga: L.S.A. 50/708).

4) ,Brief van den hertogh van Coerlandt aen sijne landtstaten ende bedroefde onderdanen, na dat hij van de Sweden na Riga gevangen is gevoert. Ende een andere uit Mitau waerin verhaelt wordt de gevangenisse van de voorz. Hertogh ende het barbarisch tractement twelck de Sweden aen Sijne onderdanen aldaer gedaen hebben". s.1. 1658.

s) Adriaan en Cornelis Lampsius, rijke kooplui uit Vlissingen, bezaten meerdere grote handelsschepen. Adriaan was een der direkteuren van de West-Indische Compagnie; zijn broer Cornelis was burgemeester van Vlissingen in 1652. $\mathrm{Zij}$ koloniseerden het eiland St. Martin en Tobago dat zij ,Nieuw-Walcheren" noemden. Cornelis ontving van Lodewijk XIV den titel van Baron van Tobago in 1663 (cf: J. Hamelton Hist. Schets der Ned. Bovenindische Antillen tot het einde der 17de eeuw; Woodstock: a history of Tobago; H. Dullaart: Histoire naturelle et morale des iles Antilles). 
profitant des troubles, qui agitaient alors la Courlande, se rendit màitre du fort, par trahison autant que par une révolution soulevée dans la garnison, sous prétexte de la restituer au Duc, dès l'instant où il serait remis en liberté. Les différents planteurs de l'île ne pouvaient pas s'y opposer. Deux ans après, le Duc fut rétabli avec sa famille dans la possession de ses états, mais la Cie Zélandaise ne songeait même pas à sa première promesse; elle tacha plutôt, par d'assez illégales meneés, d'obtenir de L.H.C. le privilège par lequel la possession de l'île de Tobago lui fût affermée. Le Duc résolut donc d'envoyer â La Haye Henri Momber 1) et Folkersamb 2) pour se plaindre des usurpations de la Compagnie de Lampsen, mais voyant ses remontrances et sollicitations sans effet, il envoya en Angleterre Fréderic de Dranckewitz, Grand Drossard de Grobinnen, comme l' instruction dateé de Mitau, le 19 mars 1660 le porte, afin de réclamer la protection du Roi d'Angleterre et d'obtenir par son intercession auprès des Etats Généraux la restitution de l'île de Tobago et un dédommagement de la Compagnie Zelandaise au Duc et à ses planteurs ${ }^{3}$ ). Mais tout ces mouvements furent inutiles et l'appui que le Roi Charles I donna aux représentations du Duc, ne produisit que de faibles paroles. Le Duc résolut donc de conclure un Traité formel avec le Roi d'Angleterre Charles II, le 17 novembre 1660, consistant en 6 Articles, dont la copie est ci- jointe ${ }^{4}$ ). Le Roi, sûrement fâché du peu d'égards que les Etats généraux avaient montré à son intercession dạs l'affaire du Duc, envoya deux vaisseaux, sous les ordres du Capt. Poole ${ }^{5}$ ) pour chasser

1) Henry Momber, geb. in 1619, overl. 1685, hoofd ener grote handelsonderneming aan de Amstel te Amsterdam, 1 Juni 1650 sloot hij met den hertog in Mittau een handelsverdrag .(cf. Mattiesen o.c. p. 98). Hij werd directeur van de hertogelijke scheepvaart.

2) Melchior Folkersamb, Kanselier van Koerland; zijn briefwisseling, die in 1939 berustte in het Staatsarchief te Riga, is waarschijnlijk verdwenen.

3) De hertog Jacobus had in Holland grote schulden gemaakt. In de Staatsarchieven te Riga (Valsts Archïvs. Fonds Kurz n. 188/1262 en 1263) liggen stapels brieven van Hollandse schuldeisers .die hem een proces willen aandoen, o.a. Witsen, koopman te Amsterdam, die 41990 gulden opeisten en Momber, aan wien de hertog 161670 gld. schuldig was. In 1715 hadden de schuldeisers hun geld nog niet ontvangen. Zie ook de correspondentie tussen den hertog en den advocaat van Burcht in het Rijksarchief in Den Haag. Liasse 1047 en 1048.

4) Zie Bijlage.

s) Capt. Poole. cf. Harlow: Barbados 1925., p. 210 vlg.; West-Ind. Gids XXII, 37; In 1672 veroverden de Engelsen onder Sir Tobias Bridges en Capt. Poole Tobago, dat in 1667 door A. Krijnsen met 25 soldaten bezet was; daarmede had Tobago opgehouden een Zeeuwse kolonie te zijn. 
les Zélandais de l'île. Le fort capitula et on y fit 500 hommes prisonniers et autant de nègres. L' année 1677. 1), l'Amiral français Comte d'Estreés, attaqua cette île avec une force de 20 vaisseaux de ligne et un grand nombre de boulets. La défense du port fut aussi vive qu'opiniâtre et soutenue avec la plus grande valeur. Le Comte d'Estrées, y perdit beaucoup de monde, son vaisseau avec 445 hommes d'equipage coula â fond; il fut blessé et se sauva avec peine dans un petit canot; mécontent de cette expédition, il se retira, en abandonnant l'entreprise; mais après avoir redoublé à l'île de Grenade, alors appartenant à la France, il renouvella l'attaque au mois d'octobre, emporta le fort de Jacobus, et ruina les plantations. Les Français ne gardèrent pas longtemps cependant cette conquête: ils l'abandonnèrent peu de temps après. La paix de Nimègue faite, le Duc, à qui l'établissement d'une colonie en Amérique, dont il a été le premier conquérant et fondateur, tenait fort à coeur envoya deréchef quelques bâtiments avec des vivres, munitions 24 pièces de canon, 2000 grenades et 20000 livres de poudre: en outre, un nombre d'ouvriers de toutes sortes de métiers, pour ériger des plantations. Le Duc se sentant favorisé par Charles II, roi d'Angleterre, envoya à Londres Abraham Morin, comme Envoyé pour se concilier la protection et l'appui du Roi, qui connaissait trop bien l'importance de cette île, si elle tombait entre les mains de la France ou de la Hollande, ou du Duc de Courlande avec lequel il avait passé un contrat formel 2).

Morin obtint le 19 février 1680 un ordre du Roi, par lequel le gouverneur des Barbados était chargé de pourvoir non seulement les vaisseaux, que le Duc y enverrait, de toutes les necessités dont ils pourraient avoir besoin, mais de les secourir en tout à condition néanmoins que ces vaisseaux s'abstiennent de quelque négoce que ce fut dans l'ile de Barbados. Le Capitaine Nagel qui partit à bord du vaisseau ,,le pot à fleurs" pour porter cet ordre, s'acquitta si mal de sa commission, que son vaisseau tomba entre les mains d'un Corsaire d'Alger, mais fut relâché quelque temps après: Ce qui engagea le Duc à se procurer un ,firman.' du Grand Seigneur, par lequel S.H. ordonnait à tous les Capitaines ou Commandeurs de son pavillon et à ceux d'Alger et d'autres corsaires sous sa protection, de respecter et laisser librement passer les vaisseaux du Duc Jacques de Courlande en tel passage qu' ils puissent être: Cet ordre ou „Firman”, magnifiquement écrit sur vélin, selon la coutume de la Sublime Porte, existe encore

1) Over den aanval van den Comte d'Estrée, zie: A. Newton: The European Nations in the West Indies 1493-1688 .p 303; C. de Jonge: Geschiedenis van het Nederlandse Zeewezen. Haarlem 1859; C. K. Kesler: Tobago (De West-Indische Gids X, 527).

2) Cf: W.-I. Gids 1940, p. 98, 99; Rotterd. Jaarboekje, 1939, p. 80. 
dans les Archives de Mitau, comme monument authentique de la considération dont le duc Jacques jouissait chez l'étranger.

Pour réparer la faute de Nagel, le Duc envoya deux autres vaisseaux, chargés de colonistes, de vivres, de munitions et ustensiles de toute espèce, sous les ordres du Colonel „,Monck” 1) qui avec l'aide des habitants de Barbados, s'etablirent de rechef à Tobago et y construisirent un nouveau fort. Le 20 octobre 1680 on fit une convention avec Jean Poyntz 2), négociant à Londres, par laquelle on lui accorda 120arpents de terre, mesure anglaise, à condition que dans l'espace de trois ans, il y ferait transporter, lui et ses compagnons 1200 personnes pour y eriger de nouvelles plantations, y bâtir un fort et des châteaux, dont ils entretiendraient les garnisons à leurs frais; que les habitants de l'île seraient exempts de tout impôt pendant sept ans consécutifs qu'après ce terme écoulé, ils payeraient deux deniers annuels pour chaque arpent en reconnaissance du fief, et qu'enfin ils auraient le libre exercice du culte divin pour toutes les religions, excepté la Catholique Romaine, et même qu'en temps de guerre, en cas qu'elle vint à exister entre le Roi d'Angleterre et le Duc de Courlande, ils demeuraient dans une parfaite neutralité.

Le Duc Jacques, quoiqu'il jouissait depuis plusieurs anneés d'une possession tranquille de l'île de Tabago, ne vit point l'exécution de ce Traité: il mourut le 31 décembre de l'année suivante: Frédéric Casimir, qui lui succéda, envoya vers $1684{ }^{3}$ ) le Land Marschall d'Alten- Bockum ${ }^{4}$ ) avec 20 hommes d'equipage à Tobago, en qualité de Gouverneur. Le vaisseau qu' il monta, éprouva une forte tempête et alla se briser contre les rochers dans le naufrage, Bockum fut blessé à la cuisse, se sauva cependant avec peine et atteignit l'île, où il mourut quelque temps après et fùt enterré.

Après sa mort, les Colonistes étaient peu encouragés et Poyntz manquant peut-être de ressources pour remplir le contrat, commenca à ralentir et à retracter. Morin, l'envoyè du Duc, vient à mourir, Frédéric-Casimir le remplace par Charles-Jean, Baron de

1) W.-I. Gids p. 104.

2) John Poyntz, geb. 1606, was een Schots koloniaal avonturier. Hij zou op verlangen van den hertog in Tobago een republiek van kolonisten in 1631 stichten. Hij was fregatten-Kapitein, later vice admiraal onder Montagise, en werd op Trinidad gevangen genomen; hij was een goed kenner der West-Indische kolonies. ( J. Poyntz: The present prospect of the island of Tobago. London 1648).; West-Indische Gids XXII p. 99.

3) In 1687 cf. W.-I. Gids 1940, 103.

4) Dietrich von Altenbockum, maarschalk, was meer officier, dan koopman en beschouwde de kolonie als een terrein van politieke en militaire activiteit. 
Blumberg, qu' il envoya â Londres au mois d' aôut 1686, pour solliciter de Jacques II la confirmation du contrat ou un dedommagement de Poyntz.

Les affaires avaient bien changé de face, comme aussi les négociations de l'envoyé du Duc. depuis la mort de Charles II. Son successeur Jacques II, fit répondre à Blumberg le 19 mai 1687 que des raisons ,,importantes" l'empêchaient de permettre qu' aucune compagnie se formât en Angleterre pour passer à l'île de Tobago et de s'y établir.

On sait avec quel zèle aveugle ce Prince était adonné au culte de l'Eglise Romaine. Un Roi qu' on a vu depuis abandonner son royaume par un excès d'enthousiasme pour cette religion, pouvait-il se résoudre â confirmer un contract par lequel cette même réligion était exclue d'une île sous sa protection? Aussi rapporte-ton qu'il eut la faiblesse de jeter le contrat aux pieds de Blumberg dans une audience particulière, en lui demandant quelle contradiction $\mathrm{y}$ avait-il à être en mème temps bon catholique et juste et sincère citoyen. Après cette scène, Blumberg retourna en Courlande sans avoir pu réussir dans sa négociation 1). Le Duc Frédéric-Casimir vient à mourir le 22 janvier 1698, et la Régence de Courlande dépêche le même Blumberg à Londres, dans le courant de la même année dont l'instruction signeé de cette Régence, est dateé de 28 mars, afin de sommer Poyntz de remplir son contrat ou d'en faire un dédommagement convenable, et à défaut de son consentement de chercher à la fin un autre entrepreneur pour conclure avec lui. Blumberg était chargé de réclamer la protection du roi Guillaume III dont les maximes fondeés sur la bonne politique, étaient bien opposées à celles de son beau-père. Arrivé à Londres Blumberg, Poyntz apauvri, la Société disperseé et peu disposeé à remplir son contrat, il en signa donc un autre le 15 Xbre de la même anneé avec Nicolas du Pin, Thomas Puckle, Richard Goddard et Joseph Blake 2). Pour la validité de ce nouveau contrat, il fallait l'aveu et le consentement du Roi, mais son Conseil de Commerce y mettait des obstacles comme à une entreprise peut être contraire aux intérêts de quelques négociants de Londres, qui avaient des vues particulières sur le commerce de l'île de Tobago. La Régence de Courlande de son côté n'y mettait point d'energie et d'assiduité pour enlever les obstacles, qui se présentaient pour conclure ce nouveau traité. L'affaire trainant de plus en plus en longeur, le Duc Ferdinand, tuteur de Frédéric Guillaume, ordonna àu commencement de l'année 1699, le 9 janvier au conseiller de la Chambre, Praetorius, de se rendre à Londres pour s'instruire du vrai état des affaires

1) W.-I. Gids 1940, p. 106.

2) Kooplieden te Londen, cf. Mattiesen o.c. p. 956. 
de Tabago: Praetorius ${ }^{1}$ ) eut déja à la Haye une audience de Guillaume III le 21 octobre et l'ayant suivi à Londres le 3 novembre de la même année, il trouva Poyntz brisé de vieillesse et d'indigence, la société de Blumberg dissoute et les membres peu au fait d'une entreprise de cette nature. Praetorius s'aperçut bientôt de l'envie et la jalousie des négociants à Londres; les chicanes et les entraves de la Régence de Courlande aussi bien que la protestation faite alors par l' Ambassadeur de France, Mr. de Jalland à Londres contre tout établissement sur l'île de Tabago, la considérant comme conquise par les armes de France sur les Hollandais, en terres de la Compagnie Zelandaise, étaient assez suffisants pour mettre de nouveaux obstacles à la négociation et à la réussite de cet objet. Sur le rapport de Praetorius au Duc, l'administrateur lui envoya ordre de transferer l'île de Tabago ou du moins une partie, à titre d' ammodiation, mais les mêmes raisons s'opposerènt à cet expédient.

Praetorius s'étant donné toutes les peines possibles d'obtenir pour son mâitre l'assistance du Roi dans cette affaire, ne put cependant parvenir qu'à recevoir communication d'une faible relation faite au Roi par le Conseil de Commerce dans laquelle on pretendait que le Duc n'avait plus de titres sur l'île de Tabago, lesquels droits cependant étaient fondés sur les droits de la première acquisition, sur coup de la nature et des gens.

On pouvait presque s'attendre à une pareille réponse, dictée par l'interêt particulier que le Conseil de Commerce y prenait. Si même l'équité de Guillaume III l'eut fait pencher pour le Duc, dont l'affaire était si juste et en dehors de toute contestation, sa politique, l' intérêt de son Royaume en montant sur le trône d'Angleterre, n'auraient pu les favoriser. Il est sûr et certain, que les véritables motifs du Conseil de Commerce étaient uniquement à faire échouer toute négociation de l'envoyé du Duc, au sujet de l'île de Tabago pour les raisons suivantes.

1. Que les Anglais ne voulaient pas, qu'une nation jusque là inconnue dans le commerce, devînt un objet de leur jalousie.

2. qu'ils craignaient pour leurs sucreries à la Barbados, si les Courlandars arrivaient à en construire aussi a Tabago.

3. Que l'île de Barbados ayant jusqu'à ce moment coupé tous les bois à Tobago, on prévoyait des obstacles pouvant s'élever

1) Praetorius in Saksen geboren, kwam op jeugdigen leeftijd naar Koerland. Hij studeerde in Den Haag, werd gezant in Brandenburg en Polen en in 1699 naar Holland en Engeland gezonden, om de kwestie „,Tabago" te regelen. Hij schreef: Tobago insulae Caraibicae in America sita fatum seu brevis et succincta insulae hujus descriptio, dat in 1727 te Groningen gedrukt werd. cf: $\mathrm{H}$. Sewigh: Eine Kurländische Kolonie (Baltische Monatschrift XXI (1872) p. 1-40. 
pour les habitants, si cette île fut entre les mains d'un mâitre qui voulût les faire valoir.

4. Qu'enfin les Hollandais, qui avaient eu l'occasion de constater les grands progrès, que le Duc Jacques avait faits dans le commerce, craignant de voir un jour la Courlande, rivale du négoce, faisaient l'impossible pour exciter la jalousie des Anglais. Il est vrai que le Duc Jacques, prince clairvoyant et très instruit dans plusieurs branches du commerce, avait 40 vaisseaux en mer, qu'il avait fait construire en Courlande, la plupart à Windau, montés de canons en fer, ayant 3 fonderies, 3 moulins à poudre, 2 de cuivre et 2 papeteries. Le Duc vendait même des vaisseaux montés de canons de fer et tout prêts à mettre à voile à l'Espagne et à la France, ayant obtenu de Louis XIV et du Parlement une permission particulière pour le commerce et l'exemption du droit d'aubaine, datée de Paris, 23 fév. 1647.

Il envoya ces bâtiments au Sund, pour y être équipés par la nation qui en faisait l'achat. Le Duc Ferdinand, le dernier de la souche de la famille de Kettler, qui avait quitté la Courlande à cause de procès continuels avec la noblesse, s'établit à Danzig et ne songeait plus à réclamer ses anciens droits sur l'île de Tobago ni à poursuivre le plan de ses ancêtres .Mais le Comte Maurice de Saxe fils naturel d'Auguste II, roi de Pologne et Electeur de Saxe, se mit sur les rangs des Prétendants pour être élu Duc de Courlande, nonobstant que le duc Ferdinand était encore en vie et n' avait pas résigné. N'ayant point de descendant et quoiqu'il eût 75 ans il se maria à une Princesse de Saxe Weissenfels. Maurice, soutenu par une faction en Pologne ,et fortifié par l'intercession de la France, trouva des adhérents en Courlande. Les Etats convoquèrent même une diète à la fin de juin 1756 à Mitau, contre le gré et la volonté de la République de Pologne et malgré la protestation du Duc Ferdinand et élirent Maurice, Comte de Saxe, duc de Courlande et de Semigalle titre, qu' il a toujours conservé en France. Tous ceux de la noblesse courlandaise qui lui étaient attachés se distinguèrent par un uniforme brun doublé de ponceau et un chapeau à plume blanche. A peine fut-il élu Duc de Courlande, qu'il songea d'abord à envoyer des colonistes à Tobago, pour y faire de nouvelles plantations à l'instar des précédents ducs et considérant cette île comme appartenant au duché de Courlande. Il en nomma gouverneur Mr. de Brincken, gentilhome Courlandais et engagea plusieurs personnes à l'accompagner, dans des conditions assez avantageuses. Il compta beaucoup, comme de raison, sur le Roi de Pologne et sur la France pour être soutenu dans cette entreprise, mais l'influence de la Cour de Russie toujours prépondérante et salutaire à la Courlande contrebalança bientôt comme de juste droit, le 
parti de Maurice, qui fut obligé de quitter la Courlande et d'abandonner son vaste projet sur l'île de Tobago.

Ernst Jean, Comte de Biron, alors Grd. Chambellan de S.M.I. l'Impératrice Anne, fut élu en 1737 Duc de Courlande et de Semgale par les Etats de ces duchés assemblés en Diète, conforme à l'ancien droit qui date du temps des Chevaliers Porte-Glaive, lesquels avaient acquis ce pays. Cette auguste Princesse sauva la Courlande du bouleversement total de sa constitution, ce que tenta la Pologne en voulant l'incorporer à la République et la partager en Palatinats et Stavosters. Le Duc Ernst Jean témoigna le désir de relever ses prétentions sur l'île de Tobago, à titre d'alleu acquis de la famille ducale de Kettler; il avait même destiné son gentilhomme de Chambre, le Baron Klopmann à être envoyé à Londres: Klopman y avait demeuré 6 ans ,après avoir été attaché à l'Ambassade du Chevalier Williams en Russie afin de solliciter le Roi, en réclamant sa protection et de renouveler l'ancien Traité, fait par le Duc Jacques de Courlande et le Roi Charles II d'Angleterre.

Par ce Traité, le Duc Jacques céda au Roi le fort St. André, sur les côtes de Guinée (ainsi que l'article 2 du Traité ci-joint le marque) aussi des plantations en Afrique témoin une lettre authentique du Prince Rupert d'Angleterre au Duc Jacques, datée du 22 mars 1652, de la rivière de Gambie lettre dans laquelle il se loue des services que le Capitaine d'un vaisseau du Duc lui a rendus dans ces parages et lui conseillant d'y faire expédier quelques vaisseaux. Aussi le Duc ne tarda-t-il pas à suivre cet avis, en y envoyant 2 bâtiments la „Prudentia” et La „Pietà”, qui y arrivèrent le 6 mai 1654 .

La mort du Duc Ernst Jean de Biron interrompit ses projets. Il est évident et hors de toute contestation possible, que l'île de Tabago a été acquise par le Duc Jacques, qu'elle a passé comme alleu par succession d'heritage dans la famille ducale de Kettler, et qu'enfin le Duc Ernst Jean en fit l'acquisition par un accommodement avec les héritiers de cette famille, comme de tous les alleux lui appartenant, en rachetant toutes les terres allodiales engagées par les Ducs précedénts, pour une somme très considérable, condition stipulée alors par le Roi et la République de Pologne, avant l'investiture du Duc.

Pierre, dernier duc de Courlande, succédant à son père Ernst Jean, résigna le 17/28 mars 1795 entre les mains bienfaisantes de l'Auguste Impératrice Cathérine II de glorieuse mémoire. 
Propositions ${ }^{1}$ ) Faites par Mr Poyntz et Cle aU DuC et SigNÉES Par Son Envoyé Mr Abraham Marin, À LondRes (copiées de l'original)

The proposals humbly offered to the Duke of Courland by John Poyntz and Cle as followeth:

1.) That if the said Duke, or any from or under him will give and grant unto the said John Poyntz and Cle one hunderd and seventy thousand acres of land in the Island of Tobago for them to enjoy in her simple for them and their heirs for ever. That then, the said John Poyntz and Cle will engage themselves to transport Twelve Hunderd persons, men, women and children; the first year 4 hunderd of the said men, women and children and the second year four hunderd and the third year, four hunderd; all with lands, the one third part to be delivered at the first arrival of the said Poyntz or any of the said Poyntz his Cle on the said island, and the second third part to be measured the second year, and the last third part to be delivered at the bringing in the full number of twelve hunderd men, women and children into the said island or to be measured out and delivered onto the said Poyntz or $\mathrm{C}^{1 \mathrm{e}}$ at the beginning of the third year, if they have delivered their said number at the said time.

2.) That if need requireth, the said Poyntz and Cle for the strengthening and peopling the said island after the first three years to engage transport or cause to be transported, the like number of people to settle, manure and maintain the said land under the said Duke in four or five years gratis and free without any claim from the said Duke, if it shall be thought fit by the governor and Assembly.

3.) That the said Poyntz and Cie do further engage, if the Governor and Assembly shall think good, will built two or more forts and maintain the same at their own costs and charges, upon the said Lands.

4.) That the said island shall be a free Port, to the said Poyntz and $\mathrm{Cle}^{\mathrm{e}}$ for seven years, without paying any taxe or toll and afterwards, to pay such taxes and tolls as shall be made and exacted by the Governor and Assembly, and the whole island to

1) Menkman in West-Ind. Gids 1940, p. 99: „In 1681 gaf hertog Jacobus aan captain John Poyntz een patent of commissie en 's hertogs vertegenwoordiger, Abraham Marin, sloot in hetzelfde jaar met Poyntz een contract". Klopmann noemt als sluitingsdatum 20 Oct. 1680. Enkele memories van Poyntz berusten op het Rotterdamse Gemeente Archief (Menkman t.a.p.)

Over deze propositions cf.: Mattiesen o.c. p. 726, die enigszins van bovenstaande verschillen. 
be a free port, to all nations, paying such impositions as shall be adjudged meet by the Governor and the Assembly.

5.) That liberty of conscience be granted to all such as shall inhabit the island - Roman Catholics excepted - provided they did nothing contrary to the laws of the Land.

6.) That all and singular the Lands so taken up as above mentioned may be entire and may begin to set down on the South East part of the said island to begin the measuring the same dividend at the Easter end, et so to run westward.

7.) That all and singular, the inhabitants may be governed by a Governor and an Assembly, which Assembly is to be chosen by the general vote of the people, double the number to be chosen, and then the Duke to name him or them that shall sit to make wholesome laws for the defence hereof annually.

8.) That no impositions or taxes shall be paid by the people, but what shall be decided by a Governor and Assembly.

9.) That after seven years possession of the said Lands, the said Poyntz and $\mathrm{C}^{1 e}$ their heirs and assignes, are to pay annually two pence per Stere as a generaly Rent to the Duke, or his Assignes for ever, as long as they shall hold the said Lands under him and upon default to distrain upon the said Land, which said two Pence per Stere shall be acknowledged by the said Duke for them to hold their Land by for ever, as a lawful purchase, and in process of time the imposition to be raised, as it has been customery in Jamaïca and the neighbouring Islands.

10.) That on oath shall be administred on to all and singular the people that belong to the said Poyntz and $\mathrm{Cle}$, their heirs and assignes and yield their alligeance to him, as above to be their lawful Prince in the said island and no other, and the administring of the oath shall be confirmed by holding up their right hand.

11.) That the Duke, his heirs and successors to choose and appoint his Governor, but all other officers to be chosen by the Governor and the Assembly.

12.) That the said Poyntz and $\mathrm{Cl}^{\mathrm{le}}$, his heirs and assignes may have free liberty to build ships, vessels or boats for their proper use, as many as they shall have occasion for, and trade with them to all parts.

13.) That a Deputy Governor and all other officers necessary to be in the said Poyntz and Cle dividend of the said island, must be appointed by the Governor and Assembly.

The above mentioned Conditions have been agreed by Iohn Poyntz (L.S.) Abraham Marin

Henry Cookman (L.S.) Thomas Poyntz (L.S.)

Hig High, the Duke of Courland Resident (L.S.) 
P.S. J'ai trouvé dans un ancient manuscript authentique, que l'Angleterre avait offert au Duc Jacques deux millions de Livres Sterling pour l'île de Tobago, connaissant l'importance d'une acquisition aussi considérable et avantageuse pour le commerce, par sa situation. Cette île produit du sucre, de la muscade, des oranges, de l'indigo, du coton, du bois de cyprès et a même la pêche des perles. Le Duc, ayant de très-grandes vues au sujet de l'acquisition de cette île qui lui tenait fort à cœur, en refusa la première offre et, par la suite, son emprisonnement par les Suédois et sa détention à Ivangorod jusqu'à la paix d'Oliva, interrompirent cette négociation, et surtout sa mort! - L'Angleterre ne perdit pas de vue son premier plan et fit pareillement négocier avec le Duc Frédéric-Casimir, au sujet de l'île de Tabago, mais toute cette négociation fut aussi interrompue et l'auteur du Manuscrit n'en marque pas les raisons, de sorte que cette cession à l'Angleterre n'a pas eu lieu, et la Maison Ducale perdit à jamais une acquisition d'aussi grande importance que l'auteur du Manuscrit soutient que tous les revenus du Duc en Courlande ne valaient pas ceux de l'Ile de Tobago.

\section{Copy of the Treaty betwixt Charles II, King of England AND JAMES, Duke OF CURLAND RELATING}

The Tobago, one of the Caribbee Islands, which was discovered, cultivated and fortified by the Duke's subjects, till they were dispossessed by the Dutch; upon which the Duke submitted it to King Charles's protection, and held it by way of grant from the Crown of England, concluded November 17th $1664^{1}$ ).

I. The King grants the Duke and his successors full liberty of Trade and Commerce for such ships, that properly belong to him and his heirs; but not those of his subjects. In any rivers or haven within the Dominions of His Majesty, on the coast of Guinea or also to trade for goods not exceeding the value of $f: 12.000$ per anno, according to the price they were first bought for, on those coasts from whench they were exported, with full liberty to build one or more store houses to lay up goods, under the castles and forts belonging to his Majesty or subjects on those coasts; to hold and enjoy the said liberties, as long as friendschip,

1) Cf. O. Mattiessen o.c. p. 601, waarin vele fouten voorkomen. Het „tractaat" van 17 Nov. 1664 is in de literatuur meermalen opgenomen of geciteerd dikwerf met vele fouten; een copie berust in het Rotterdamse Gemeente-Archief (stukken betreffende Tobago 1664, 1684, 1698) cf. W.I. Gids l.c.p. 34 noot 1. Zie verder: J. J. Danxion Lavaysse: Voyages autour des iles de Trinidad, de Tobago, etc. II; Calendar of States Papers, America and West India 1661, etc. 
amity and a good understanding continued between the said King and the Duke.

II. In consideration of the above mentioned concession and grant, the Duke makes over to His Majesty, his Heirs and the Successors the fort of $\mathrm{S}^{\mathrm{t}}$ Andrew in Guinea and all the forts and fortifications or sconces there belonging to the said Duke together with all guns, bullets and powder and other instruments of war there to belonging.

III. The Duke agrees that himself and his heirs shall pay $3 \mathrm{p}$. for all goods imported or exported from any of his Majesty's ports in Guinea.

IV. Moreover His Majesty grants to the said Duke and his heirs the Isle of Tobago, together with all Lands, havens, creeks, rivers and profits to the same belonging, to be held and enjoyed under the King's protection. Provided always that the said Duke shall not suffer any other besides his own subjects and the subjects of His Majesty to abide in the Island, to settle plantations or build houses, but the King's Subjects shall have all the privileges of the Duke's subjects.

V. The Duke moreover agrees and promises, that neither he, nor his heirs, nor his subjects nor any for their use, shall export any merchandise or products of the said Island of Tobago, or import otherwise than out of or into some ports belonging to England and Courland or the ports of the city of Dantzig.

VI. Moreover for the sake of acknowledging, that the Duke of Courland does hold and enjoy the said island from His Majesty it is further provided and agreed, that when and as often the King of Great Britain, and his heirs shall require it, or when he or they happen to be engaged in a war against any other King, Prince or State except the King of Poland, the Duke of Courland, at their own proper charge from time to time, shall bring or cause to be brought one good man of war, furnished with 40 great iron guns, to such ports, station or place, as His said Majesty shall name; into which ship his Majesty shall put commanders and seemen and supply them with victuals and wages, under the conduct and at the expense of his said Majesty, so long as the said ship shall continue in his or their service, which shall never be more than a year at one time.

In testimony and for the undoubted confirmation of this Treaty, Charles, King of England and James, Duke of Courland have signed and sealed the above named Treaty November $17^{\text {th }}$ 1664.

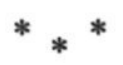

Olivarus Protector Reipublicae Angliae, Scotiae, and Hiberniae omnibusque et singulis Nostris admiralibus, Thalassiarchis Gene- 
ralibus Praefectis, Tribunis, Capitanis, nec non omnibus et singulis Nostris Legatis, Residentibus aliisque quibuscunque quorum interest per presentes significamus quod cum Serenissimus Princeps, Dominus Iacobus in Livonia, Curlandia et Semigallia Dux, quo populis et dictionibus sibi subjectis, de quiete et tranquillitate prospiciat et contra metuendas futurorum motuum calamitates tempestiva immunitatis remedia quaerat ${ }^{1}$ ). Nos per generosum virum Johannem Bretislaw Mislik, Baronem ab Hirstorf, ablegatum ad Nos, suum rogaverit. Ipsi nostram salvam guardiam, a cunctis belli incommodis plenissimam exceptionem et neutralitatem in solemni et amplissima forma concederemus. Nos igitur commoti justitia et aequitate hujus sui desiderii hanc petitam salvam guardiam, exceptionem et neutralitatem libenter ei concessimus. Mandamus itaque et volumus quod omnes et singuli sub Nostrae Republicae signis belli gerentes cujuscunque conditionis et dignitatis sint praedictum Principem dominum Iacobum, in Livoniae, Curlandiae et Semigalliae Ducem, cum omnibus ducatibus territoriis et dominiis suis, aliisque inde dependentibus, omnibusque subditis et incolis, absque infestatione vivere et agere, incolasque et subditos, sua commercia terra marique, libere exercere permittant, atque illos ut amicos ubique habeant et hac nostra salva guardia citra noxam et incommoda ubique perfrui omnimode concedant, sub pœna indignationis Nostrae. Mandamus insuper omnibus et singulis Nostris Legatis, Residentibus et Deputatis aliisque Ministris, publicis jam constitutis vel inposterum constituendis ut oblata omni occasione justa et honesta, dicto Principi ac Domino Iacobo, in Livonia, Curl. et Semigal. Duci, rogati, in manutenda hac Salva Guardia, ejusque contentis maturis, authoritateque et prudentia prosint. Denique mandamus et hoc Nostrum prescriptum in copiïs vidimatis cum supra dicti Principis literis Salvi Conductus subditis suis concessis exhibitum, pari cum Autographo et originali authoritāte et fide gaudeat. In quorum omnium et singulorum fidem Nostras patentes propria manu signavimus; iisque magnum Sigillum Angliae apponi fecimus. Dabantur ex aula Nostra Westmonasterii, vigesimo octavo die Augusti anno Domini supra millesimum sexcentesimum quinquagesimo quarto.

\section{De la part du Roi ${ }^{2}$ )}

A notre très-cher et bien-aimé cousin le Duc de Beaufort, Pair, Grand Maître, Chef et Surintendant Général de la navigation et du Commerce de France, et à tous Gouverneurs et nos Lieute-

1) cf. Mattiessen o.c. p. 395.

$\left.{ }^{2}\right)$ cf. Mattiesen o.c. p. 612 . 
nants Généraux en nos provinces et armées, Capitaines et Gouverneurs de nos villes, et places maritimes et autres chefs d'escadres, capitaines et conducteurs de nos vaisseaux et galères, baillifs, Sénéchaux, Prévôts, Juges ou leurs Lieutenants, maires et echevins de nos dites villes, gardes des ports d'icelles et de nos ponts péagers et passagers et à tout autre de nos officiers et sujets qu'il appartiendra,

Salut.

Nous voulons et vous mandons très-expressément, que vous ayez à laisser sûrement et librement passer et repasser par tous les lieux et endroits de vos pouvoirs et juridiction les navires appartenant à notre très-cher et bien aimé Cousin, le Duc de Courlande et de Semigalle, sans leur donner ni à ceux de leurs équipages aucun arrêt, trouble ou empêchement, mais, au contraire, toute l'aide, faveur et assistance dont ils auront besoin; à la charge, toutefois, qu'ils ne porteront aucune marchandise de contrebande et d'autant que des présentes on peut avoir besoin en divers endroits.

Nous voulons qu'aux copies d'icelle, dûment collationnées foi soit ajoutée comme au présent original, car tel est notre plaisir.

Donné à Fontainebleau, le $23^{\text {lme }}$ jour de Juin mil six cent soixante six.

\section{Mémoire du Duc de Courlande présenté au Roi de France}

Au Roi,

Le Duc de Courlande est obligé de représenter très-humblement à Votre Majesté que les Etats qu'il possède ne relèvent ni de l'Empereur, ni d'aucun autre Prince; il les a seulement volontairement mis sous la protection du Roi et de la République de Pologne. Il possède bien quelques terres situées sous la domination de l'Empereur, des Rois d'Espagne, d'Anglettere, de Suède et de Danemark, à lui concédées par les dits-Rois, mais dans la présente guerre il n'a pris part pour aucun des Princes et Etats qui y sont engagés, s'étant toujours particulièrement attaché à la Pologne et à ceux qui sont en paix et bonne intelligence avec elle. Il a eu soin surtout, de mériter l'honneur des bonnes grâces de V. M. C'est pourquoi ayant su que V. M. trouvait mauvais que deux de ses fils fussent au service des Hollandais (ce qu'ils ont fait sans affection de parti, mais seulement pour apprendre le métier de la guerre), Son Altesse les a aussitôt rappelés; l'un a incontinent obéi et est retourné au pays, l'autre ayant différé plus longtemps, S. A. l'a déshérité ,de quoi ce fils a conçu un si grand déplaisir qu'il en est mort. Quant au régiment qui porte le nom de Courlande, à cause de son défunt colonel, il est composé de Prussiens, Polonais, Allemans, Suédois et d'autres nations, le plus 
petit nombre est de Courlande et vu la neutralité en laquelle est Son Altesse, les Suédois font des services dans les états avec toute liberté. - La bonté de V. M., envers S. A. le Duc de Courlande a passé en ce que par un traité fait en 1643, ratifié et registré en nos parlements, la Chambre des Comptes de Paris, V. M. a permis à $\mathrm{S}$. A. et à ses sujets la liberté de commerce dans tous les ports de France, avec exemption du droit d'aubaine pour ses sujets et de plus a permis à la dite Altesse d'acquérir et de posséder des châteaux et terres en France, avec pareille exemption du droit d'aubaine, de sorte qu'après la mort de S. A. les dits biens seraient conservés et appartiendraient à ses héritiers.

En outre de cela, V. M. a encore, en l'an 1666, accordé un passeport général, pour tous les navires et biens de sa dite Altesse et de ses sujets.

D'ailleurs, V. M. a eu la bonté de s'obliger à la garantie de la paix d'Olive, conclue avec la Suède en 1660 et a promis à S. A. de lui faire restituer les sommes qui lui sont dûes par le Roi de Suède et les meubles, vaisseaux et autres choses qui lui ont été enlevées pendant la guerre finie par le dit Traité de Paix avec des terres acquises par Son Altesse et qui depuis 17 à 18 ans sont détenues par des particuliers de la ville de Riga, sans que le Roi de Suède en retire aucun avantage et ces terres sont exposées aux courses et invasions des Moscovites s'il y avait guerre entre eux et la Suède, ce qui fait voir la grande nécessité que S. A. a de rentrer en possession des dites terres, parce qu'elle ne pourrait guère les retirer des Moscovites, s'ils s'en étaient emparés. Non obstant, et au prejudice de ce qu'en outre les navires français ont pris plusieurs navires de S. A. dont il n'a pu obtenir la restitution ${ }^{1}$ ).

Le premier, nommé ,,la Mère de Courlande”. Cpt. Leckt partit dans le courant du mois de Décembre 1670 (plus de 15 mois avant le commencement de la présente guerre), pour l'île de Tobago, frêté aux frais de S. A. qui en est en possession sous la garantie du Roi d'Angleterre dès l'an 1654, car ce navire, ayant été jeté pa la tempête sur les côtes de Granada, il fut obligé d'atterrir, nor pas pour négocier, mais pour y prendre de l'eau, ce qui fut fait avec la permission du Gouverneur; néanmoins, sous prétexte que le dit Leckt, capitaine Courlandais fit cadeau en partant de deux barils de bœuf à un navire français qui manquait de vivres, le dit navire Courlandais a été emmené et déclaré de bonne prise, à la Martinique, sous ce seul prétexte, qu'il avait déchargé du bœu fà Granada malgré et contre la défense de V. M. qui ne veut pas que d'autres que les Français trafiquent dans ces îles.

Le second navire, nommé ,l'Orpheus", allant à Rotterdam a été pris par un armateur de Dunkerque où le dit navire a été

1) W.I. Gids l.c. p. 35 . 
mené. - Le troisième, nommé „La Rose” destiné pareillement pour Tobago, a été pris et mené à Brest. — Le quatrième, nommé „l'Island" qui devait aller premièrement à Newcastle, en Angleterre et de là à Tobago, a été pris près du Sund et mené aussi à Dunquerque. De tous ces navires appartenant à S. A., on n'a pas pu obtenir la restitution, quoique S. A. croit n'avoir point été contraire à vos ordonnances, ni rien fait qui puisse avoir donné lieu à la prise et adjudication des dits navires.

$\mathrm{S}$. A. supplie encore très-humblement V. M. de se souvenir que du temps de feu Mgr le Cardinal Mazarin, S. A. équipa à ses frais vingt quatre navires pour le service de V. M. sans que S. A. ait été remboursée des frais de l'équipage, ni de l'entretien des dits navires, qui demeurèrent assez longtemps sur ses coffres. Il a été dit ci-dessus que depuis l'an 1654 et sous la garantie du Roi d'Angleterre, S. A. est en possession de l'île de Tobago, qu'elle a fait défrischer et y a fait bâtir des forteresses et il en a joui paisiblement jusqu'à ce que les Suédois ayant fait prisonniers le Prince, la Princesse et toute sa famille à Mitau, un rebelle nommé Lambson, Hollandais qui avait été établi dans la dite île, persuada à ceux de la garnison que $\mathrm{S}$. A. ne serait jamais relachée, et les fit se résoudre à se vendre aux Hollandais, sur qui V. M. l'avait prise depuis peu.

Toutes ces choses, Sire, obligent S. A. de s'adresser à V. M. et de redoubler ses prierès et très-humbles supplications à ce qu'il lui plaise, par grâce et bonté, de faire droit à S. A. sur tous les chefs ci dessus:

1. Premièrement, qu'il plaise à V. M. de renouveler et confirmer à S. A. ses agents, facteurs et sujets la liberté de trafiquer dans tous les ports de France, avec exemption des droits d'aubaine, conformément au traité de 1643, ratifié et depuis registré en Votre Parlement et en la Chambre des Comptes.

2. Deuxièmement, Renouveler et confirmer aussi le passeport général accordé par V. M. en 1666 à S. A. pour tous ses navires et ceux de ses sujets, pour aller partout où bon leur semblera.

3. Troisièmement, Faire payer à S. A. les frais de l'équipage et l'entretien des 24 navires équipés pour la service de V. M. durant le ministère de feu Son Eminence, le Cardinal Mazarin.

4. Quatrièmement, Faire rendre aussi les dits quatre navires la Mere „l'Orpheus”, ,,la Rose” et „l'Islande”, menés à Dunkerque et à Brest ensembles avec les marchandises dont ils étaient chargés avec dépens, dommages et intérêts.

5. Cinquièmement: Faire pareillement rendre à S. A. les forts et terres qui lui appartiennent dans l'île de Tobago.

6. Sixièmement: Conformément et en exécution du Traité d'Olive, de l'an 1660, obliger les Suédois à rendre à Son Altesse les terres qu'ils lui détiennent, l'argent, les vaisseaux, les meubles et autres choses qu'ils lui ont prises, lesquelles ils sont obligés de 
rendre par le dit Traité, à la garantie duquel V. M. a eu la bonté de s'obliger. Enfin, et si V. M. ne voulait pas faire rendre ou payer les dits quatre vaisseaux la Mère, l'Orpheus, La Rose et l'Islande menés à Dunkerque et à Brest, ni payer en argent les frais de l'équipage et entretien des 24 navires mis à son service, V. M. aura la bonté, s'il lui plait d'accorder à S. A. la propriété incontestable de quelques terres bâties en France, pour en jouir par S. A., sa postérité et ligne, avec exemption du droit d'Aubaine et autres droits reversibles, ainsi que l'Empereur et les Rois d'Espagne, d'Angleterre, de Suède et de Danemark en ont accordé de semblables à la dite Altesse laquelle proteste de persister toujours dans le respect et service de V. M.

D'un autre côté, S. A. offre à V. M. de lui fournir du goudron et autres denrées de son pays, à meilleur marché qu'on ne peut les avoir ailleurs.

Donation du Roi d'Angleterre au Duc Guillaume de Courlande:

Iacobus Dei gratia, Angliae, Scotiae, Franciae, Hiberniae Rex, Fidei Defensor, etc., etc.

Omnibus ad quos praesentes hae pervenient, salutem. Cum multis certissimis argumentis Nobis sit exploratum Illustrissimum Guilhelmum in Livonia, Curlandia, Semigallia Ducem, consanguineum nostrum perdilectum, singulari studio et obsequio ita nobis esse addictum vt propter affinitatis et cognationis necessitudinem ipsius officia, et merita majorem in modum nostram illi gratiam et benevolentiam concilient. Cumque non solum, ad honorem Regni nostri conducat, sed etiam praesidii non nihil in eo sit ut apud ceteras gentes, illustres et principes viros, nobilitate ac virtute praecellentes, Nobis liberalitate et beneficientia nostra devinctos habeamus, visum est Nobis, praedictum Consanguineum nostrum ob eas, quos antea diximus, consanguinitatis nobilitatis virtutis, ac praesertim singularis ergo nos studii, et obsequii causas, honorario donare stipendio animumque ipsius sponte sua Nobis addictum beneficio etiam devincire.

Sciatis igitur, quod Nos de gratia nostra speciali, ac ex ista scientia, et mero motu nostro, ac praeantedictis causis, et considerationibus, nos ad praesens specialiter moventibus dedimus et concessimus, ac per praesentes pro Nobis Haeredibus et Successoribus nostris concedimus praedicto Duci Guilhelmo, Consanguineo nostro quondam annuitatem sive pensionem quadringentarum librarum, bonae et legatis monetae angliae, per annum summam bis mille thalerorum exeguentium, habendam et annuatim percipiendam, eandem annuitatem, sive pensionem quadringentarum librarum per annum praefato Duci Guilhelmo, et assignatis suis, à festo Sancti Michaelis Archangeli ultimo ante datum 


\section{DE KOERLANDSE KOLONISATIEPOGINGEN OP TOBAGO}

harum literarum nostrarum patentium ad terminum ac pro durante termino vitae naturalis, ipsius Domini Guilhelmi, de thesauro nostro, haeredum et successorum Nostrorum, ad receptum Successoris Nostri heredis, et successorum Nostrorum, per manus Thesaurarii et Camerarii nostrarum heredum et Successorum Nostrorum ibidem pro tempore existentium, ad quatuor anni terminos usuales videlicet ad Festa Natalis Domini,Annonciationis beatae Virginis Mariae, Nativitatis Johannis Baptistae, et Sancti Michaelis Archangeli, per aequales portiones solvendas. Eo quod expressa mentio de vere valore annuo, vel de certitudine premissorum, sive eorum alicujus, aut de aliis donis, sive concessionibus per nos seu per aliquem progenitorum sive praecessorum nostrorum praefato Domino Guilhelmo ante haec tempora factis in praesentibus facta existet. Aliquo statuto, actu, ordinatione, provisione sive restitutione, in contrarium, inde ante haec factis editis, ordinatis sive praemio aut aliqua alia re causa, vel materia quacumque in reliquo non obstante. In cujus rei testimonium has literas nostras fieri fecimus patentes teste me ipso Westminster, Octavo die Octobris Anno Regis Nostri Angliae, Franciae et Hiberniae quarto et Scotiae quadragesimo, sub Nostro privato sigillo.

(L. S.) 